\title{
0 INPA e sua Biblioteca: uma síntese histórica
}

\author{
Algenir Ferraz Suano da Silva (") \\ M. Arlete de Jesus Harraquian (")
}

\begin{abstract}
Resumo
Histórico da Biblioteca do INPA, de sua fundação até o presente, focalizando suas atividades, metodologia de trabalho e meios de acesso às informaçōes. Mostra ainda a organização de suas coleções bibliográficas, o planejamento em biblioteconomia, modo de utilização, preparação e arquivo das informaçōes. No final inclui lista de todas as publicaçōes editadas pelo INPA.
\end{abstract}

\section{INTRODUÇÃO}

A Biblioteca do INPA é um Centro Dinâmico de Documentação e Informação, mantendo organizada a crescente coleção de obras de todas as áreas do conhecimento humano, destacando-se Ciências Puras e Ciências Aplicadas. Diariamente, novas publicações são recebidas pela Biblioteca e incorporadas ao seu acervo para que os usuários possam utilizar o vivo material bibliográfico.

Atualmente este Órgão ocupa 3 pavilhões destinados, respectivamente:

a) Auxílio aos leitores, com salas para leitura em grupo (12 lugares), estudo em conjunto (60 lugares) e oito (8) gabinetes individuais;

b) Acervo;

c) Processos Técnicos, que compreende 11 salas: duplicatas, desenho, fotografia, reprografia, diretoria, secretaria e processos técnicos de preparação de material bibliográfico, Setor de Acta Amazonica e Sala de Seminário (com capacidade para 70 pessoas) .

A Biblioteca do INPA colocou à disposição dos estudiosos de todas as categorias - pesquisadores, alunos e professores - que aqui exercem suas atividades e aos que se acham fora da região ou do país, um precioso manancial de conhecimentos impressos e não impres- sos. Possuidora que é de um acervo de valor inestimável, não só quanto ao aspecto científico, mas também quanto ao material, tal como livros editados desde a data de 1698 (Chridtopher D'Acungna-Voyages and discoveries in South America) até a mais moderna reprodução bibliográfica - as microformas - a Biblioteca vai se impondo na comunidade cultural do Amazonas por sua inequívoca utilidade e importância.

\section{EVOLUÇÃO HISTORICA}

Em julho de 1954, com a posse do primeiro Diretor do INPA, Dr. Olympio Ribeiro da Fonseca Filho, a Biblioteca tinha sua instalação assegurada.

Em 09 de novembro do mesmo ano foi registrado o seu primeiro livro lconografia de Orchidaceas do Brasil de F. C. Hoehne, 1949.

Organizada pela professora Dores de Queiroz Carvalho, em 1955, foi continuada até o presente pela professora Algenir Ferraz Suano da Silva.

Inicialmente, quando a direção geral do INPA ocupava o prédio da rua Simão Bolivar, a Biblioteca foi instalada no $10^{\circ}$ andar do Edifício do IAPTEC, à Avenida 7 de Setembro $n$ ? 121, onde junto com o Setor de Estatística, ocupava uma única sala. Em 1956, foi instalada no prédio $n^{\circ} 116$ da rua Guilherme Moreira, sede provisória do INPA, onde ocupava o $2^{\circ}$ andar e atendia ao público precariamente, considerando que o mesmo era bastante numeroso.

Em 1973, passou para a sede própria do INPA, no Aleixo, ocupando, de início, cinco salas do Pavilhão de Patologia Tropical. Em 1974 instalou-se definitivamente no Pavilhão próprio, numa área de $962 \mathrm{~m}^{2}$ (Fig. 1). Em 1975. recebeu uma estrutura metálica para ampliar

(") - Instituto Nacional de Pesquisas da Amazônia, Manaus. 


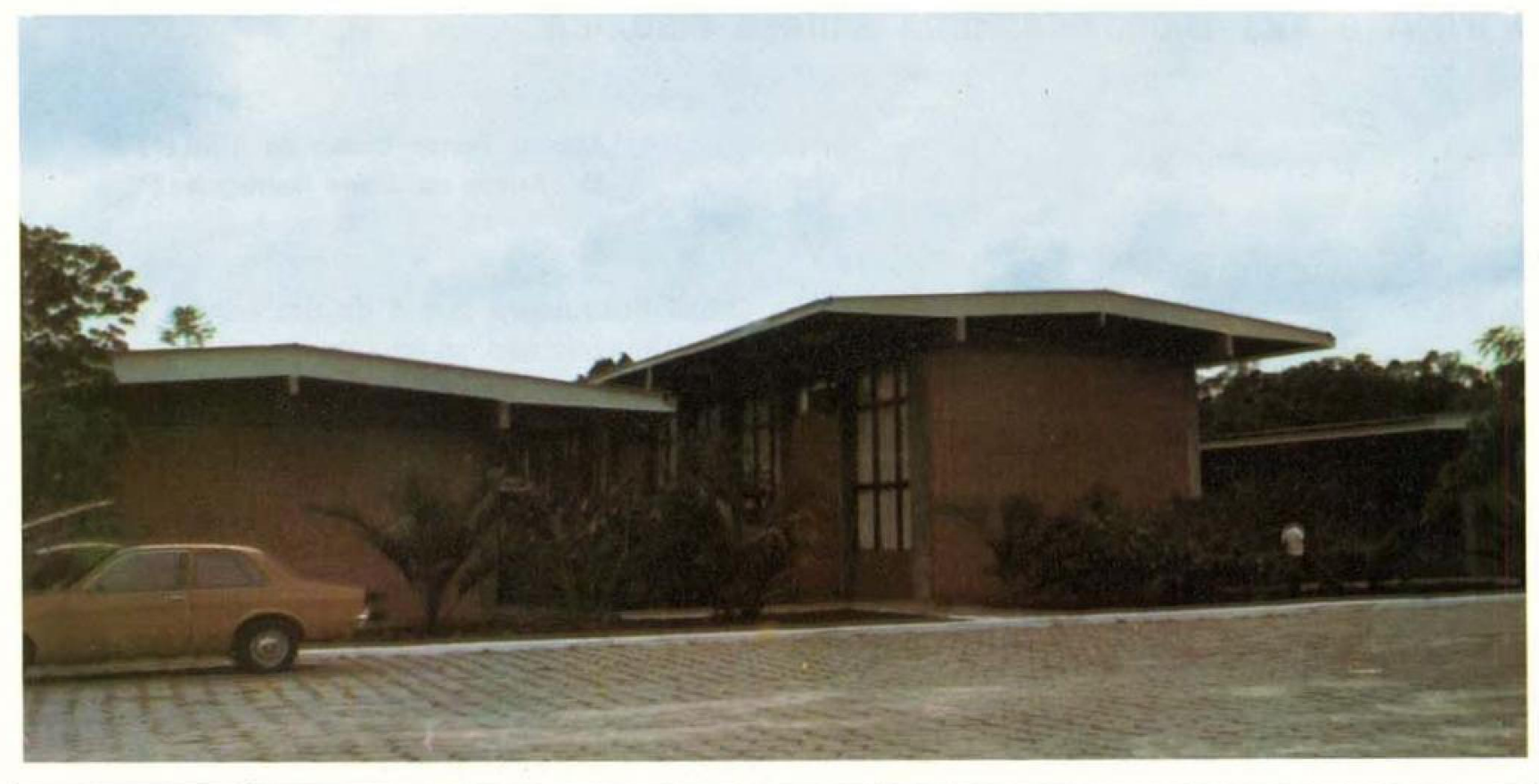

Fig. 1 - A fachada da ärea ocupada pela Biblioteca, vendo-se os três Pavilhões.

a capacidade do acervo e com uma grande verba, esse acervo foi realmente duplicado (Fig. 2 e 3).

A Biblioteca do INPA reveste-se de particular importância não só em virtude do isolamento e tranquilidade que se propicia aos pesquisadores do Instituto, mas também por considerar-se principal fonte de consulta à disposição da mocidade estudantil e dos interessados em assuntos culturais da região, num raio de $2.500 \mathrm{~km}$ (INPA, 1978) (Fig. 4) .

A Biblioteca do INPA possuî em seu acervo a maior bibliografia da Amazônia, chegando aproximadamente a 8.000 títulos. Entre os grandes autores da coleção amazônica, destacamos as obras clássicas de Wallace, Bates, Darwin, Spruce, La Condamine, D'Orbigny, Coudreau, Spix e Martius, Agassiz, Goeldi e seus colaboradores, Hoehne, Schomburgk, Humboldt, Koch-Grünberg, Keller, Barbosa Rodrigues, Le Cointe, Alexandre Rodrigues Ferreira e outros. Em A Biologia Central Americana, considerada entre outros uma das coleções de História Natural mais rara do mundo em seus 64 volumes pintados à mão, encontramos na parte entomológica, uma das maiores obras de arte já vistas. Ainda encontramos em seu acervo as obras que pertenceram à Biblioteca do Museu Botânicơ de Barbosa Rodrigues que, por intermédio de um convênio entre o INPA e o Colégio Estadual do Amazonas, foram depositadas na Biblioteca deste Instituto. Entre as raridades destacamos a História Natural de Buffon, em 94 volumes, Linnaea, Journal für die Botanik, considerada muito rara; a Paxton's Magazine of Botany, também pintada à mão e com revestimento dourado, é considerada uma preciosidade.

Foram incorporadas, por compra, em 1976 as bibliotecas particulares dos professores $\mathrm{Nu}$ nes Pereira e Felisberto Camargo.

Em 1979, esta Biblioteca, recebeu como doação da Smithsonian Institution, através de a professora Calderón, a maior e mais completa bibliografia sobre gramíneas, principalmente os bambus, o que tornou esta Biblioteca a mais capacitada para dar qualquer informação sobre o assunto.

Existe no acervo da Biblioteca, já atingindo a dinâmica do mundo atual, uma considerável coleção de microfilmes e outros tipos de materiais especiais, como mapas, cassetes etc.

\section{ACERVO}

O atual acervo da Biblioteca soma 178.140 peças bibliográficas, englobando livros, separatas, folhetos, fascículos de revistas, cassetes, mapas, microfilmes etc. 
O crescimento da Biblioteca foi possível graças a permutas com instituições nacionais e estrangeiras, aquisiçōes, algumas assinaturas concedidas a titulo de cortesia e outras financiadas pelo Projeto BID (Fig. 5) .

\section{ORÇAMENTO}

Nos exercícios de 1954 a 1980, foram investidos Cr\$ 2.797.570,96 (Dois milhões, setecentos e noventa e sete mil, quinhentos e setenta cruzeiros e noventa e seis centavos) na renovação de assinaturas e novas aquisições. O gráfico dá uma idéia do esforço crescente do INPA, no sentido de manter atualizada e ampliar a sua Biblioteca. Cumpre notar que os recursos orçamentários da Biblioteca são sempre tirados de outros projetos, o que faz com que esta esteja sempre nos projetos de todos os Departamentos do INPA (Fig. 6).

\section{CONSULTAS}

O número total de consulentes atendidos de 1956 a 1980, atingiu a cifra de 396.568. De-

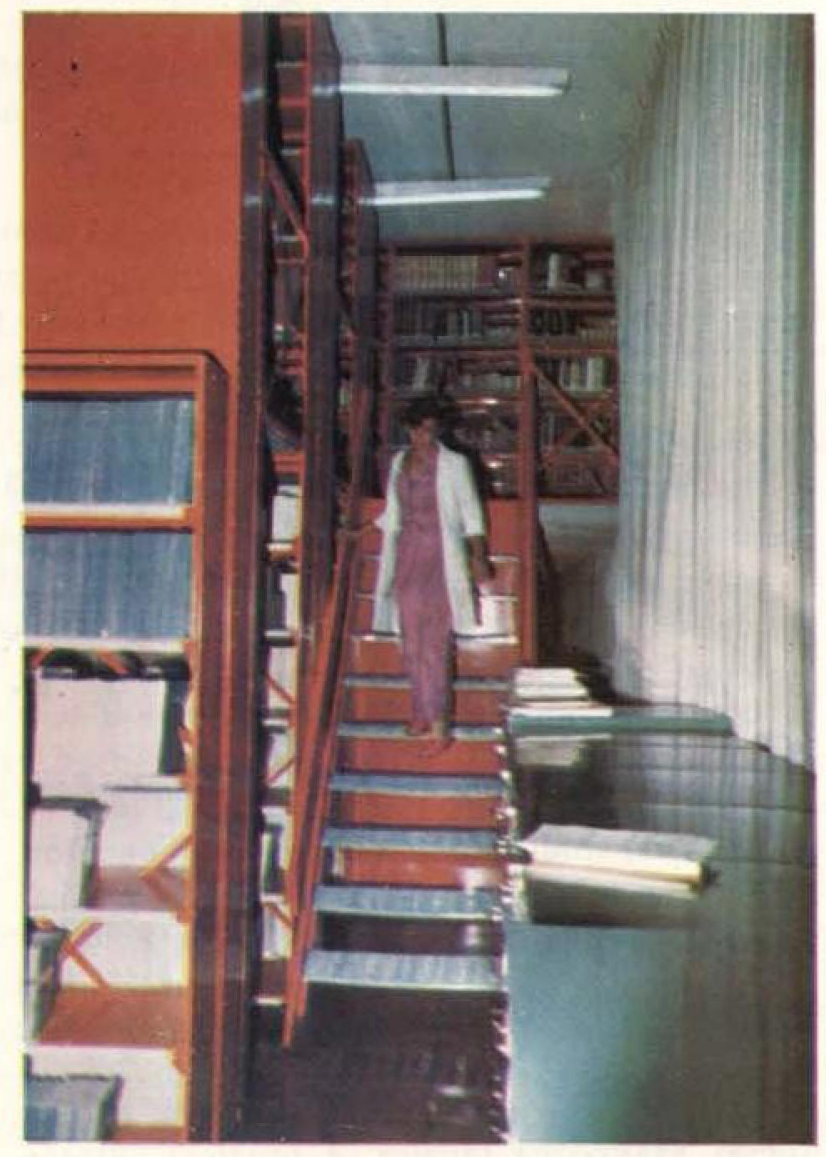

Fig. 2 - Pavilhão do Acervo, parte interna, aparecendo a estrutura metálica de dois pisos.

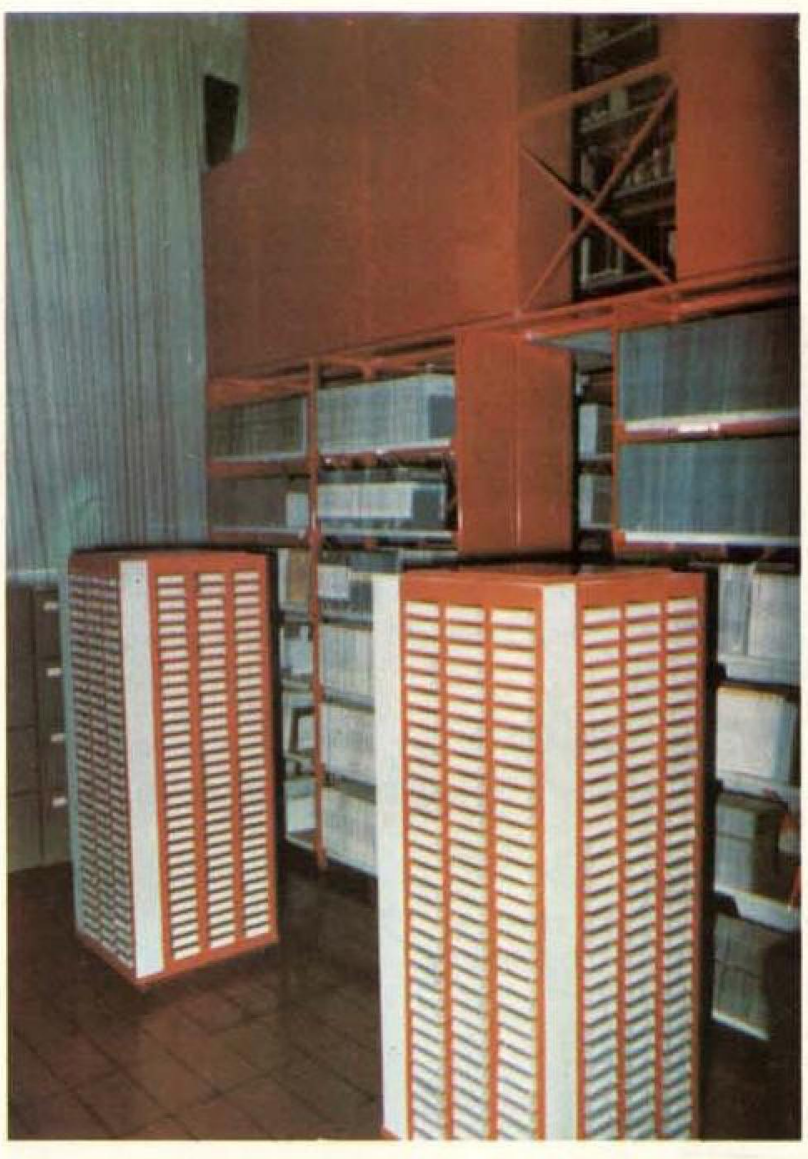

Fig. 3 - Pavilhăo do Acervo parte interna, aparecendo os escaninhos de microfilmes.

vemos notar que, até 1973 , só eram computadas as consultas de universitários e de pessoa! de nivel superior. De 1974 até a época atual são computadas as consultas de primeiro e segundo graus, pois, pela falta de Bibliotecas locais, o INPA foi obrigado a franquear suas obras especializadas a todos os estudantes de Manaus.

O número de consulentes atendidos (Fig. 7) foi considerável, levando-se em conta a distância e a localização das unidades educacionais que ficam quase todas no centro urbano de Manaus, e ao fato de que atualmente as Bibliotecas das unidades da Universidade do Amazonas estão muito bem aparelhadas e com ótimo desempenho.

A cifra do gráfico é altamente honrosa para os estudantes de Manaus e significa responsabilidade extra para a Biblioteca do INPA. 


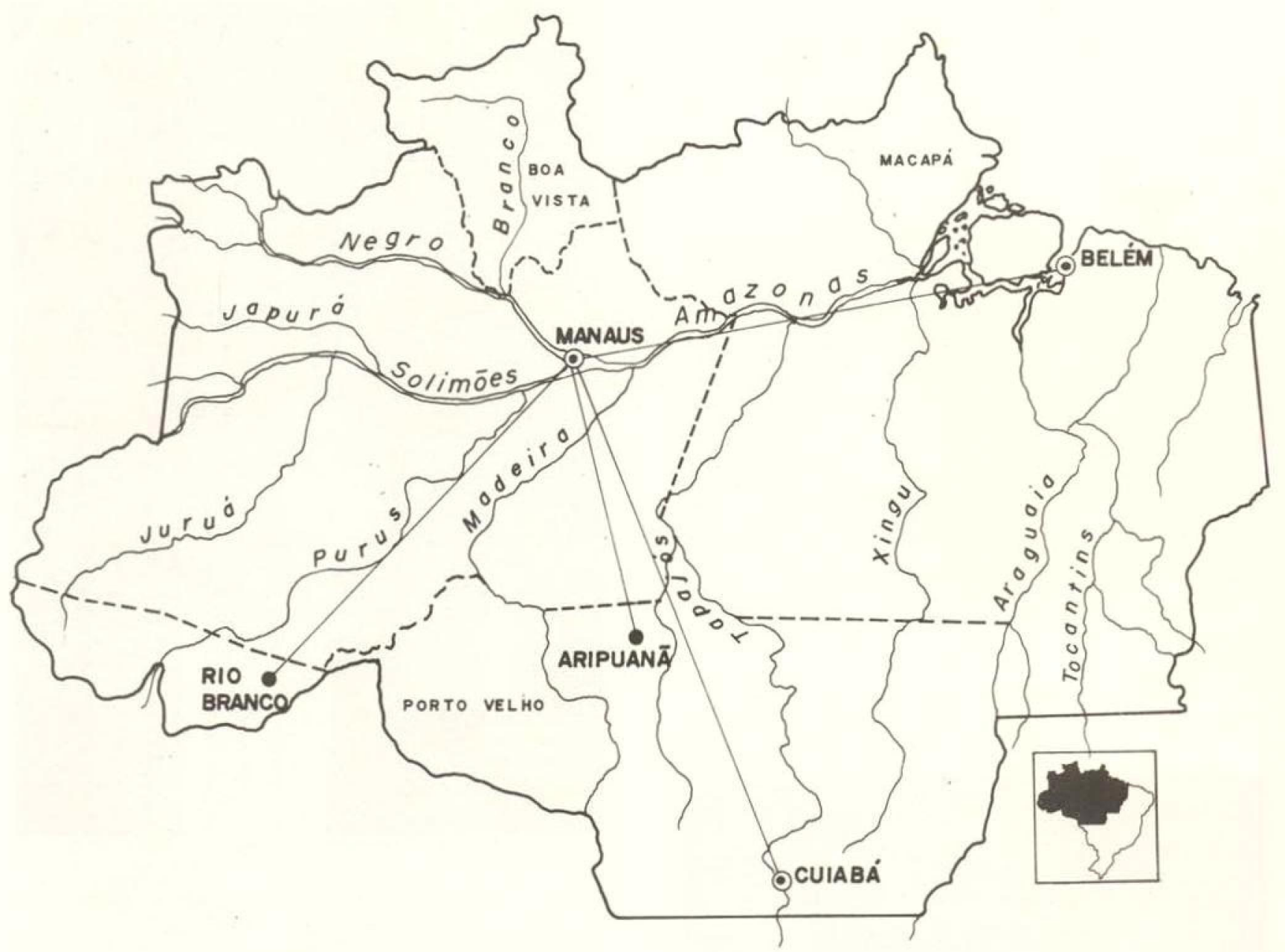

Fig. 4 - Mapa da Amazônia Legal, onde se encontram localizadas as Bibliotecas da Região que fazem parte do CNPq.

\section{OBJETIVOS}

A Biblioteca do INPA, como toda Biblioteca, tem como objetivo propiciar um sistema capaz de coletar, analisar, processar tecnicamente, armazenar, recuperar e disseminar as informações para as atividades que buscam o aperfeiçoamento e o desenvolvimento cultural dos técnicos e usuários do INPA, dentro do contexto da realidade amazônica,

Para que o objetivo seja atingido, compete à Biblioteca :

a) Selecionar e adquirir material bibliográfico adequado para o uso dos pesquisadores e técnicos da regiäo;

b) Normalizar e facilitar o uso e a distribuição de informações para as atividades de pesquisas, permitindo o intercâmbio entre instituições; c) Dotar a Biblioteca de uma infra-estrutura de instalações, equipamentos, materiais e serviços devidamente operacionalizados para atender às necessidades de pesquisas do ensino e treinamento do desenvolvimento tecnológico regional a nível nacional e internacional;

d) Adoção de nova tecnologia de informações;

e) A compatibilização de um sistema de informações para o desenvolvimento da Amazônia.

\section{ESTRUTURA}

Atualmente, a Biblioteca do INPA tem 1 (uma) assessoria técnica, 4 (quatro) setores técnicos e (três) setores de auxilio administrativo (Fig. 8). 


\begin{tabular}{|c|c|c|c|c|c|c|}
\hline ANO & LIVROS & FOLHETOS & PERIÓOICOS & $\begin{array}{l}\text { MATERIAIS } \\
\text { ESPECIAIS }\end{array}$ & TOTAL & $\begin{array}{c}{[0 / 1]} \\
\text { concimperto }\end{array}$ \\
\hline 1954 & 430 & 45 & - & - & 475 & - \\
\hline 1955 & 837 & 235 & - & - & 1.072 & 55,69 \\
\hline 1956 & 1.461 & 500 & - & - & 1.961 & 45,33 \\
\hline 1957 & 2.441 & 679 & 2. 104 & - & 5.224 & 62.46 \\
\hline 1958 & 5.411 & 860 & 3. 250 & $=$ & 9.521 & 45,13 \\
\hline 1959 & 8. 232 & 1.168 & 4. 296 & - & 13.696 & 30,48 \\
\hline 1960 & 10.991 & 1.477 & 5.620 & - & 18.088 & 24,28 \\
\hline 1961 & 13.968 & 1.938 & 7.142 & $=$ & 23.048 & 21,52 \\
\hline 1962 & 14.602 & 2.671 & 9.143 & * & 26,416 & 12,74 \\
\hline 1963 & 15,061 & 3.537 & 11.374 & - & 29.972 & 11,86 \\
\hline 1964 & 15.940 & 4.743 & 14.401 & - & 35.084 & 14,57 \\
\hline 1965 & 16.767 & 6.525 & 16.755 & - & 40.047 & 12,39 \\
\hline 1966 & 17.229 & 7.855 & 20.575 & - & 45.659 & 12,29 \\
\hline 1967 & 17.497 & 8.059 & 24.608 & - & 50,164 & 8,98 \\
\hline 1968 & 17.897 & 8.416 & 29.411 & - & 55.724 & 9,97 \\
\hline 1969 & 18.133 & 9.188 & 32.551 & . & 59,872 & 6,92 \\
\hline 1970 & 19.025 & 9.794 & 36.816 & . & 65.635 & 8,78 \\
\hline $197 !$ & 19,344 & 10.651 & 45.319 & - & 75.314 & 12,85 \\
\hline 1972 & 19.649 & 11.406 & 50.780 & - & 81.895 & 8,03 \\
\hline 1973 & 20.074 & 12.108 & 58.121 & . & 90,303 & 9,31 \\
\hline 1974 & 20.402 & $12.69 \mid$ & 60.739 & - & 94.032 & 3,96 \\
\hline 1975 & 22.071 & 15.694 & 65331 & 5. 827 & 108.923 & 13,67 \\
\hline 1976 & 25.614 & 19. 366 & 72.593 & 6. 287 & 122.860 & 11.34 \\
\hline 1977 & 26.719 & 21.472 & 79.660 & 6.354 & 134.205 & 8,45 \\
\hline 1978 & 28.421 & 27.678 & 86.797 & 7,126 & 150.022 & 10,54 \\
\hline 1979 & 30,642 & 32.136 & 94.351 & 7.500 & 164.637 & 8,87 \\
\hline 1980 & 32.755 & 36.431 & 100.576 & 8. 378 & 178.140 & 7.57 \\
\hline
\end{tabular}

MÉOIA DE CRESCIMENTO ANUAL = $17.69 \%$

Fig. 5 - Quadro demonstrativo do Acervo Bibliográfico.

Assessoria Técnica - É constituída pelos pesquisadores INPA que acompanham os trabaIhos de Classificação do Material Bibliográfico.

Setor de Processos Técnicos - Compreende os serviços de Seleção, Aquisição, Registro, Classificação, Preparação do material para Empréstimo e Conservação das Estantes, bem como, Catálogos.

Setor de Tratamento Técnico de Materiais Especiais - Composto pelos serviços de preparação de Periódicos, Recortes, Mapas, Microfilmes, Microfichas, Filmes Curtos, Fitas Gravadas, Separatas, Slides e Discos.

Setor de Auxílio aos Leitores - Compõese de Serviços de Referência, Orientação aos Leitores, Circulação, Publicidade e Bibliografia.

Setor de Acta Amazonica - Compete normalizar e publicar a produção bibliográfica do INPA, bem como, tratar de sua distribuição. A Biblioteca tem ainda a seu encargo a publicação da Revista do INPA Acta Amazonica, cujo primeiro número (abril de 1971) substituiu as publicações anteriores. Inicialmente surgiu $\sigma$ impresso intitulado Publicações: Série Antro- pologia, Botânica, Cadernos da Amazônia, Medicina, Química, Avulsa e Viagem Filosófica.

Em 1970, o Diretor na época, Dr. Paulo de Almeida Machado, alterou o título para Boletim do INPA, englobando os estudos de Pesquisas Florestais, Patologia Tropical, Botânica e Tecnologia.

Finalmente, o nome e o formato da publicação sofreram modificações, passando à denominação atual, hoje publicada trimestralmente e reune todos os trabalhos realizados nos diversos Setores do INPA.

O crescimento do número de trabalhos publicados pelo INPA em Manaus, está ilustrado em gráfico (Fig. 9).

Setores de Auxilio Administrativo - Secretaria, Desenho, Reprografia, Fotografia, to-

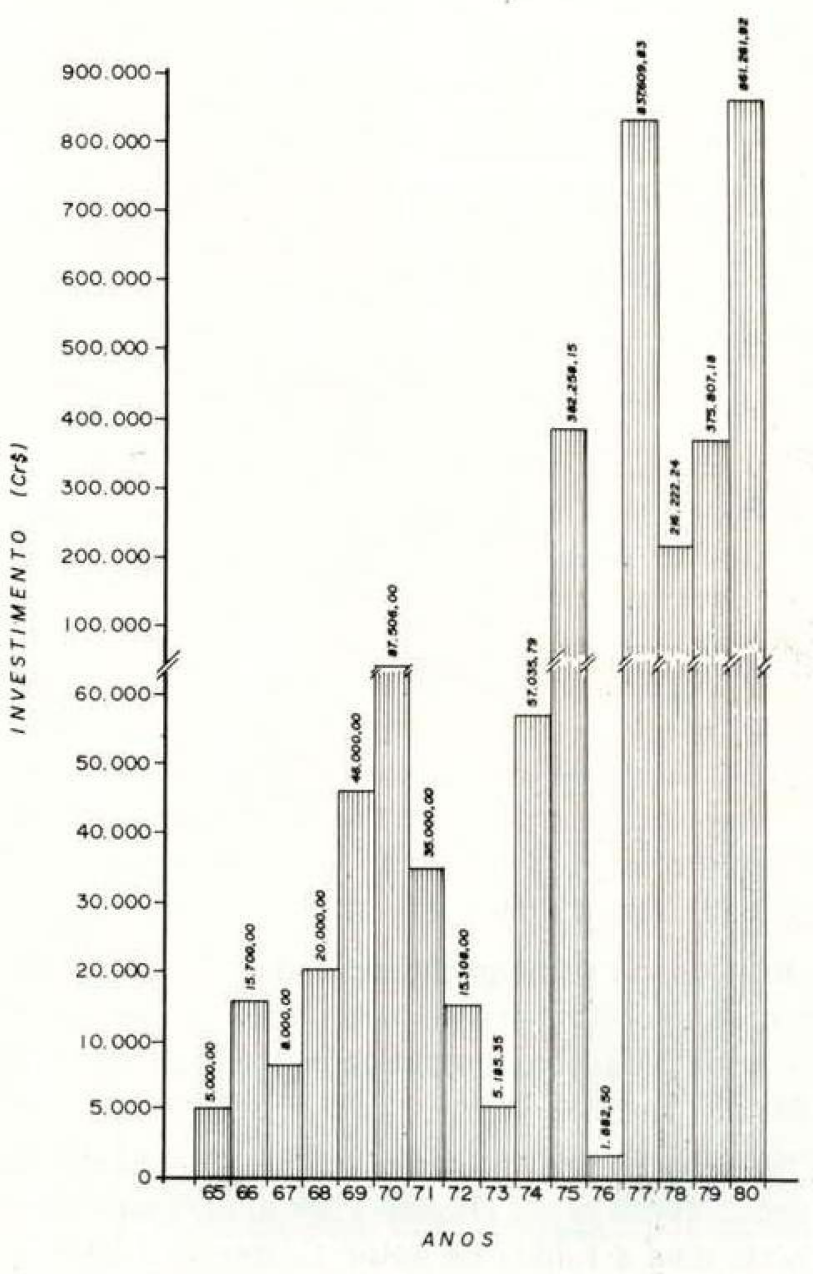

Fig. 6 - Gráfico de investimento anual com material bibliográfico no período $1965 / 1980$. 


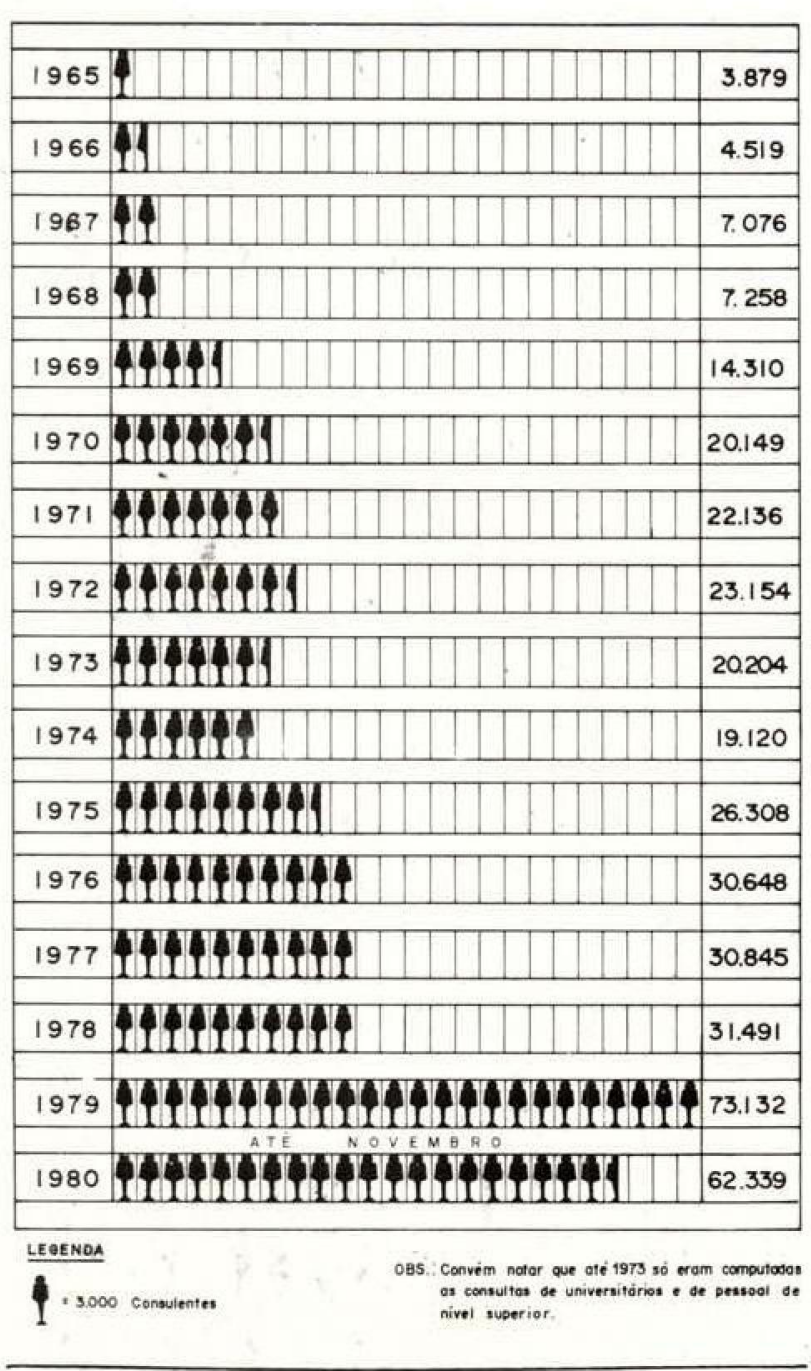

Fig. 7- Gráfico com número de consulentes atendidos pela Biblioteca, computados de 1965/80.

dos com responsabilidades especificas. Os citados Setores atendem à Biblioteca, bem como executam atividades de todo o Instituto, o que faz considerá-los Setores operantes. O Laboratório de Fotografia foi transferido para a Biblioteca do INPA em 1971.

Normas da catalogação adotadas - Catalogação Anglo Americano para casos específicos e a Catalogação Simplificada para os demais casos.

Sistema de classificação adotado - Classificação Decimal de Dewey $\left(18^{\circ}\right.$ e $\left.19 .^{\circ}\right)$ em conjunto com a Tabela de autor, Cutter - Sanborn.

No Setor de Auxílio acs Leitores, encontramos ótimas acomodações e um bom Sistema de Atendimento (Fig. 10) .
Acesso às publicações (usuários) - Os leitores tem livre acesso ao catálogo geral, onde encontram as fichas de todas as publicações existentes na Biblioteca, podendo usá-las no local sem vigilância de qualquer natureza de $2^{\text {a }}$ a $6^{\text {a }}$ feiras das 7:00 às 24:00 horas. Entretanto. se desejar tomar uma publicação emprestada, deverá levá-la ao balcão de circulação e assinar a ficha de empréstimo que será retirada como comprovante do mesmo (Norma da Biblioteca).

\section{CATALOGOS EXISTENTES}

Para os livros, teses, folhetos e outro qualquer tipo de material bibliográfico, o catálogo geral, é dividido em :

a) Autores individuais e corporativos;

b) Títulos;

c) Assunto.

No Catálogo de Autores, são adicionadas as fichas de tradutores, Editores e de Colaboradores.

As fichas de Autores são alfabetadas pelos sobrenomes, seguido dos prenomes, de acordo com as regras de catalogação adotadas.

As Teses recebem o mesmo tratamento dos livros e suas fichas são arquivadas no mesmo catálogo dos livros, entretanto, o seu armazenamento é em separado.

\section{PERIÓDICOS}

O Catálogo usado é o Kardex que lista alfabeticamente os títulos correntes e os descontinuados existentes na Biblioteca. A Biblioteca também possui o Catálogo impresso dos periódicos listados no Kardex e sua atualização é feita através do Boletim Informativo Bibliográfico, quinzenal, publicado pela Biblioteca.

\section{MATERIAIS ESPECIAIS}

Os Materiais Especiais: Microfilmes, Microfichas, Mapas, fitas Gravadas, Cassetes, Filmes Curtos e Recortes de Jornais, que têm suas fichas arquivadas no mesmo catálogo e da mesma maneira que os materiais. Contudo, as cores das mesmas diferem, de acordo com o tipo de material. 


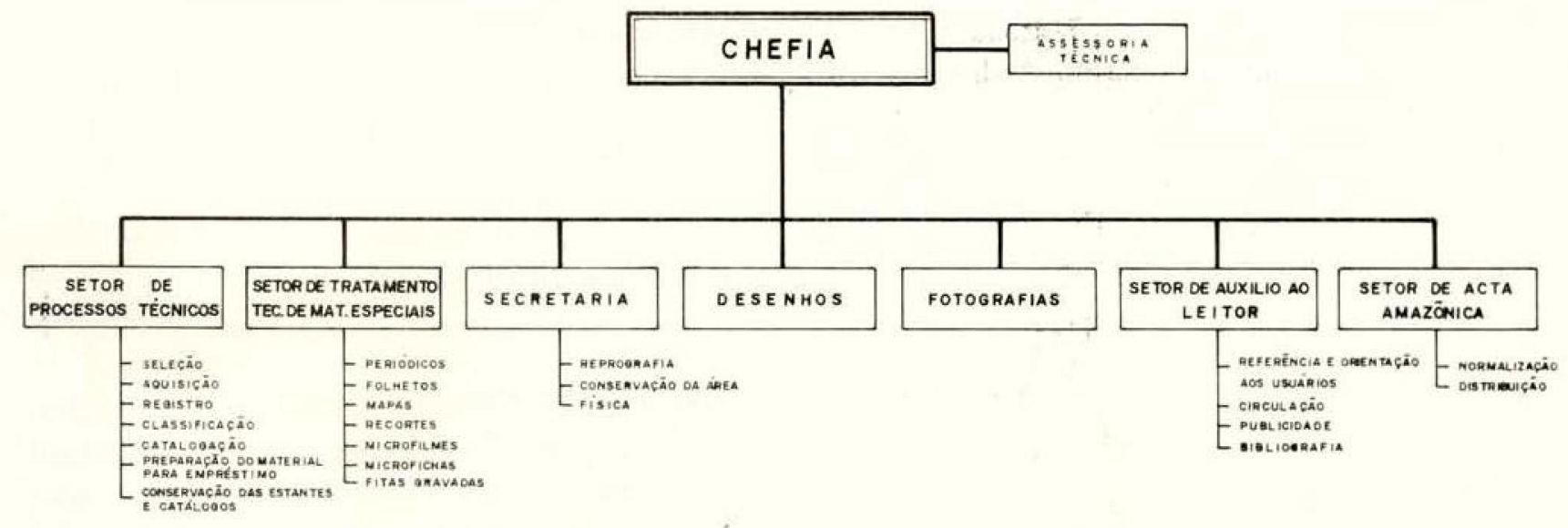

Fig. 8 - Organograma Estrutural da Biblioteca - 1980 .

\begin{tabular}{|c|c|c|}
\hline Microfilmes & - & ficha azul \\
\hline Microfichas & & ficha verde \\
\hline Recortes & 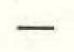 & ficha amarela \\
\hline Mapas & - & ficha rosa \\
\hline Fitas Gravadas & - & ficha laranja \\
\hline
\end{tabular}

\section{COLEÇÃO DE REFERÊNCIA}

Encontram-se no Pavilhão de Auxílio aos Leitores, e, é considerada pelos especialistas como uma grande coleção. Na Referência, o usuário encontra o Larouse do século XVII, a Britânica, Enciclopédias, Dicionários e na parte especializada, existem: Chemical Abstrats, Biological Abstracts, Index Medicus (Cumulated), Abstracts of Mycology, Zoological Record, Chemical Titles, Analytical Abstracts, Genetics Abstracts, Plant Breeding Abstracts, Helminthological Abstracts, Protozoological Abstracts, Entomology Abstracts, Abstracts on tropical Agriculture, Florestry Abstracts. Estas obras não são emprestadas e as consultas se limitam apenas ao recinto da Biblioteca.

\section{RECURSOS HUMANOS}

Dispõe a Biblioteca de 1 Técnico de Informação, 9 Bibliotecários, 6 Auxiliares de Administração, 5 Datilógrafos, 1 Operador de gráfica, 1 Desenhista, 1 Fotógrafo, 1 Assister e Técnico e 3 Auxiliares de Serviços Gerais. Devemos notar que a Biblioteca funciona com 3 turnos.

\section{Técnico de Informação}

Algenir Ferraz Suano da Silva

\section{Bibliotecários}

Alcinéa Fernandes Nogueira

Francisca Inês do Rocha Barros

Ivonete Liberato da Silva

Julieta da Costa Lima

Maria Arlete de Jesus Harraquian

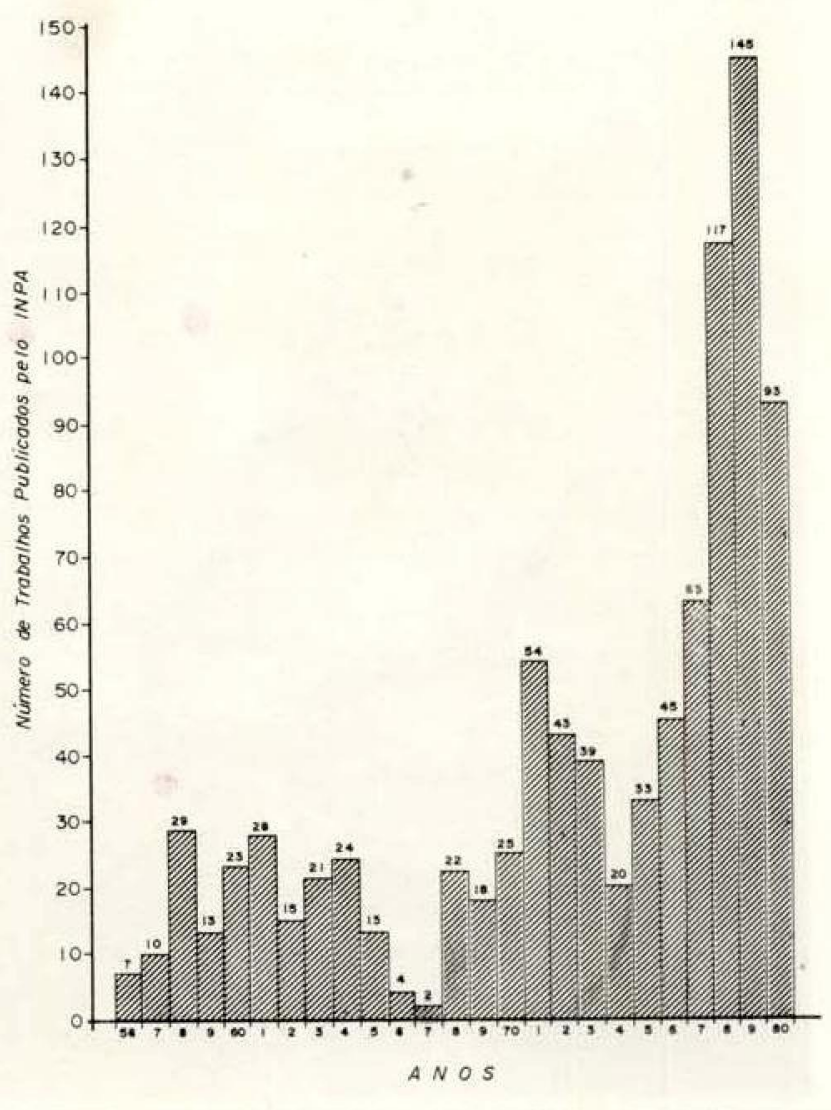

Fig. 9 - Gráfico das publicações editadas pelo INPA de $1956 / 80$. 
Maria Lenir Oran Fonseca Feitosa Maria de Lourdes D' Avila de Andrade Rosemery Leite Correia Sandra Maria Bisi Nicolau

\section{Auxiliares de Administração}

Alba Soares Pereira

Eurijader de Oliveira Veras

João Bosco da Silva Lima

Maria Helena do Nascimento Barcellar Terezinha Ferreira Mesquita

Waldizia Paula de Lima

\section{Datilógrafos}

Idalécia Ennes Vara

Maria Auxiliadora de Lima Santos

Maria Eliana Nascimento Ferreira

Maria de Matias Fernandes Silva

Wanderly Diniz de Carvalho

\section{Operador de Gráfica}

Marlindo Sagratzky de Oliveira

\section{Desenhista}

Jorge Soares Dácio

\section{Fotógrafo}

Eduardo Vieira da Silva

\section{Assistente Técnico}

João Crhysostomo de Oliveira

\section{Auxiliares de Serviços Gerais}

Álvaro dos Santos Nogueira

Ivanete de Souza Rebouças

Mariano Lira dos Anjos

\section{EQUIPAMENTOS DISPONIVEIS}

Duas xerox 7000

Um Gravador Eletrônico

Um Mimeógrafo Elétrico

Uma Mini-Graph

Um Adress Graph

Uma Plastificadora

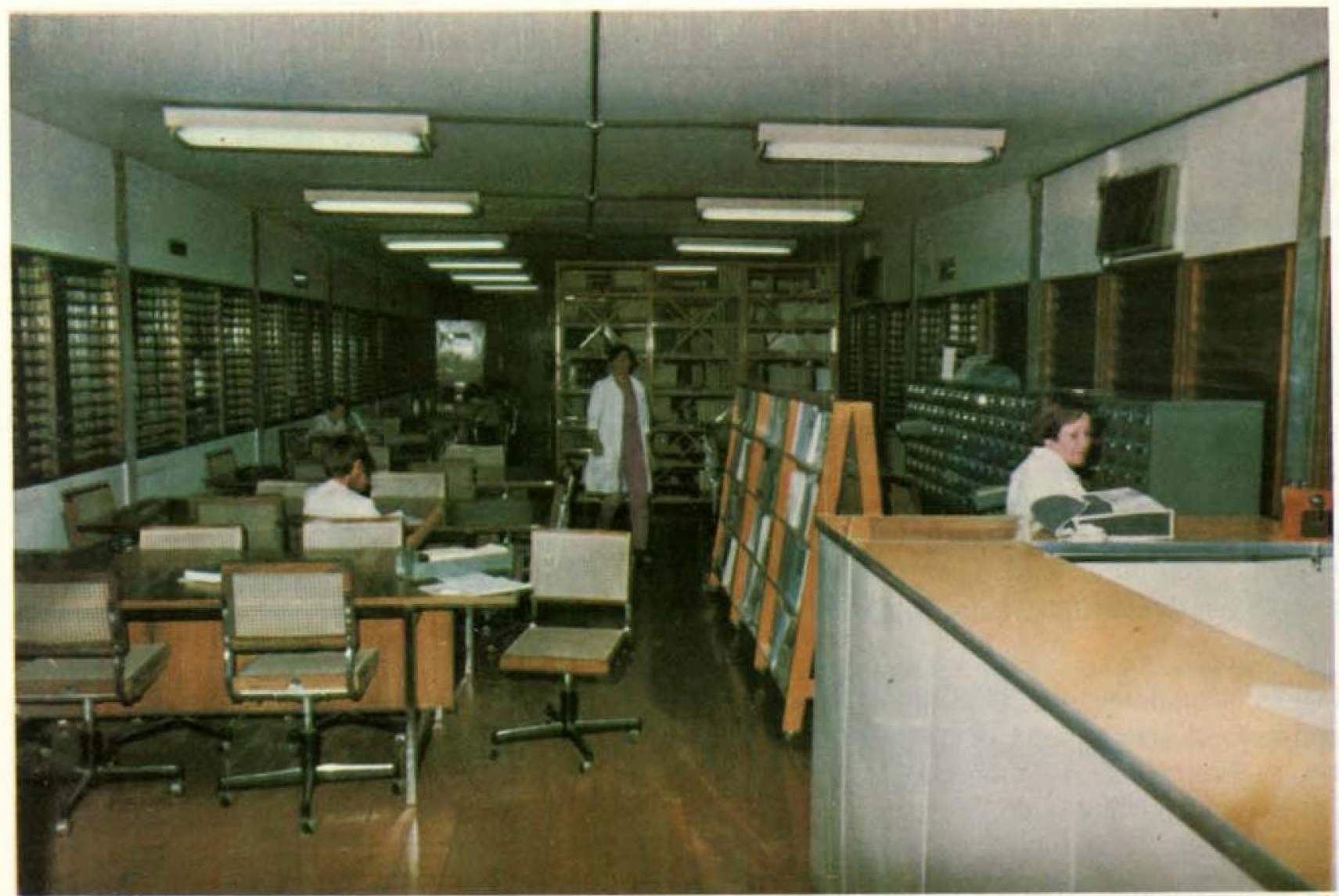

Fig. 10 - Setor de Auxílio aos Leitores. 
Uma Encadernadora (espiral)

Uma Leitora e Copiadora de Microfilmes

Duas Leitoras de Microfichas

Dois Gravadores.

Esse material representa significativa con quista para melhor atender aos usuários da Biblioteca.

A Biblioteca do INPA, coopera de maneira relevante, com outros Centros e Bibliotecas Nacionais, enviando Boletim Informativo de todo material bibliográfico (quando solicitado) . Coopera ainda com os Centros Nacionais de Documentação - IBICT, DID/EMBRAPA e com as redes de Bibliotecas da Amazônia, e, podemos afirmar, com todas as Bibliotecas brasileiras e estrangeiras que dela se utilizam para solicitar dados bibliográficos ou outras quaisquer informações.

\section{SUMMARY}

A brief history of the INPA Library, since its foundation up to the present, is being shown here, its activities, working methodology and ways of getting information. It also showns the organization of its book collections the plamning in Library Techology. ways of utilization, preparation and filing of information. Finally, it includes a list of all INPA publications.

\section{TRABALHOS PUBLICADOS PELO INPA}

PUBLICAÇÃO DO INPA: Série Antropologia

1. MONTEIRO, M.Y. - Memória sobre a cerâmica popular do Manaquiri. Rio de Janeiro, 1957.

PUBLICAÇÃO DO INPA: Série Avulsa

1. SANTOS, H.M. - Balanço hídrico de Manaus, Amazonas. Manaus, 1968.

PUBLICAC̣ĀO DO INPA: Série Botânica; 1956 - 1969

1. ARENS, Karl - Fungos no côco babaçu. Rio de Janeiro, 1956 ,

2. ARENS, Karl - Sobre a anatomia da semente do guaraná. Rio de Janeiro, 1956.

3. LECHTHALER, R. - Inventários das árvores de um hectare de terra firme da zona "Reserva Florestal Ducke. Municipio de Manaus. Rio de Janeiro, 1956.

4. ARENS, Karl - Histometria quantitativa de madeiras. Rio de Janeiro, 1957.

5. KUHLMANN, J.C. - Novitates floras amazônicas. Rio de Janeiro, 1957.

6. ARENS, Karl - Estudo anátomo-histológico Ja madeira de açacu visando o seu aproveitamento para a fabricaçăo de celulose. Rio de Janeiro, 1958.

7. ARENS, Karl - Contribuição para o estudo farmacognóstico Pluchea suaveolens (Vell.) O. Kuntze. Rio de Janeiro, 1958.

8. BRADE, A.C. - Melastomatáceas novas da Região Amazônica. Rio de Janeiro, 1958.

9. RODRIGUES, W.A. - Lista dos nomes vernáculos da flora do Território do Rio Branco. Manaus, 1959.

10. RODRIGUES, W.A. - Estudo preliminar de mata de várzea alta de uma ilha do baixo Rio Negro, de solo argiloso e úmido. Manaus, 1961.
11. RODRIGUES, W.A. - Contribuiçăo ao estudo da flora Amazônica. 1 Castanha Curupira. Manaus, 1961.

12. RODRIGUES, W.A. - Ensaios preliminares de germinação de castanha curupira em laboratório (Nota prévia). Manaus, 1961.

13. RODRIGUES, W.A. - Estudo preliminar de duas áreas de ocorrência de "Castanha Curupira". Manaus, 1961.

14. RODRIGUES, W.A. - Árvore hapaxanta na floflora Amazônica. Manaus, 1962.

15. RODRIGUES, W.A. - Estudo anatômico da madeira de Castanha Curupira. Manaus, 1962.

16. RODRIGUES, W.A. - Ocorrência de troncos ôcos em mata de baixio da regiāo de $\mathrm{Ma}$ naus, Amazonas (estudo preliminar). Manaus, 1964 .

17. RODRIGUES, W.A. - Uma nova sabiácea na Amazônia. Manaus, 1964.

18. MAINIERI, C. - Madeiras de Symphonia globulifera L., Platonia insignis Mart., Moronobea coccinea Aubl. e Moronobea pulchra Ducke (Guttiferae): Estudo anatômico macra e mi . croscópico como contribuiçăo para sua identificaçāo. Manaus, 1964.

19. MAINIERI, C. - Contribuiçāo ao estudo ana tômico do lenho de parahancornia da Amszônia. Manaus, 1954.

20. COIMBRA FILHO, A.F. - Bibliografia florestal brasileira (1:" contribuiçăo). Manaus, 1964.

21. BASTOS, A de Miranda - Contribuiçāo ao conhecimento da floresta Amazônica (Duzen tos nomes novos de árvores da Amazônia). Manaus, 1964.

22. PAULA, J.E. de - Notas sobre a anatomia de Clusia grandiflora Splitg (Guttferae). Manaus. 1966. 
23. ARAÚJO, V.C. de - Sobre a germinação do Aniba (Lauraceae). I. Aniba duckei Kostermans (Pau-rosa itaúba). Manaus, 1967.

24. LOUREIRO, Arthur A. - Contribuição ao estudo anatômico de Croton lanjouwensis (Muell. arg.) Jablonsk e Croton matourensis Aublet (Euphorbiaceae). Manaus, 1968.

25. RODRIGUES, W.A. - Duas novas espécies da flora Amazônica. 1) Novo Dicypellium Lauraceae da Amazônia. Manaus, 1968.

26. FALCÃO, J.I. - Duas novas espécies da flora Amazônica. 2) Uma nova espécie de Convolvulaceae da Amazônia. Manaus, 1968.

27. ALBUQUERQUE, B.W.P. de - Contribuiçāo ao conhecimento de Aspidosperma album (Vahl) $R$. Ben. e Aspidosperma obscurinervium azambuja da Amazônia (Apocynaceae). Manaus, 1968.

28. ALBUQUERQUE, B.W.P. de - Novas Rutaceae da Amazônia. Manaus, 1968.

29. SILVA, M.F. da - Estudos sobre Caryocaraceae. I. Contribuição para o conhecimento da morfologia foliar de Caryocar glabrum (Aubl.) Pers. e Caryocar microcarpum Ducke da Amazônia. Manaus, 1968.

PUBLICAC̣ÃO DO INPA: Série Medicina; 1958

1. BATISTA, Djalma - Resultados do tratamento dispensarial, especialmente em dois grupos: comunicantes e "caboclos". Rio de Janeiro, 1958.

2. BATISTA, Djalma - Cadastro torácico e morbidade tuberculosa. Rio de Janeiro, 1958.

PUBLICAC̣ÃO DO INPA: Série Química; 1958 - 1968

1. ALTMAN, R.F.A. - A presença da oioibina na "Catuaba" (Ponteria sp.). Rio de Janeiro, 1956.

2. ALTMAN, R.F.A. - A presença de escatol no "Tachy preto" (Tachigalia myrmecophila, Ducke). Rio de Janeiro, 1958.

3. ALTMAN, R.F.A. - A presença de Santonina no "Caxinguba" (Ficus anthelmintica, Mart.). Nota preliminar. Rio de Janeiro, 1958.

4. ALTMAN, R.F.A. - A exploração industrial de sementes oleaginosas amazônicas. Rio de Janeiro, 1958.

5. ALTMAN, R.F.A. - A industrialização do fruto do buriti (Mauritia vinifera Mart. ou M. flexuosa). Manaus, 1964.

6 MARAVALHAS, Nelson - Cinco estudos sobre a farinha de mandioca. Manaus, 1964.

7. LIRA, M.B. - Bromatologia da farinha de mandioca produzida no Amazonas. Manaus, 1964.

8. ALTMAN, R.F.A. - Estudos sobre o fruto de "umari" (Poraqueiba paraensis, Ducke e P. sericea, Tul.). 1) A composição química do fruto de "mari". (Nota preliminar). 2) O óleo do fruto de "umari". Manaus, 1965.
9. ALTMAN, R.F.A. - Duas oleaginosas da Amazônia. 1) O óleo de "andiroba" (Goniodiscus elaeospermus).

MARAVALHAS, N. - Duas oleaginosas da Amazônia. 2) "Castanha de pendula" (Couepia longipendula Pilg.) - Valor econômico. Manaus, 1965.

10. MARAVALHAS, Nelson - Estudos sobre o guaraná e outras plantas produtoras de cafeina. Manaus, 1965.

11. ROCHA, A.I. da - A presença de palmatina na Abuta grandifolia. Manaus, 1967.

12. ROCHA, A.I. da - A presença de alcaloides em espécies botânicas da Amazônia. Manaus, 1968.

PUBLICAÇÃO DO INPA: Série Viagem Filosćfica

1. FONSECA, O. de O.R. da - Alexandre Rodrigues Ferreira: o patólogo. Rio de Janeiro. 1958.

2. ANDRADE, A. de - Alexandre Rodrigues Ferreira e a introdução do método científico nos estudos sociais do Brasil. Rio de Janeiro, 1958.

3. ALEXANDRE Rodrigues Ferreira na visão de quatro naturalistas no Museu Nacional. Rio de Janeiro, 1958.

PUBLICAÇĂO DO INPA: Série Avulsa (Mimeografada)

1. ALTMAN, R.F.A. - O leite de "assacu ou Arvore do Diabo" (Hura crepitans, L.) (Nota preliminar). Manaus, 1956.

2. LECHTHALER, R. - Sobre o problema de aproveitamento racional das madeiras da Amazônia. (Nota preliminar). Manaus, 1956.

3. REIS, A.C.F. - Os índios da Amazônia. Manaus, 1956.

4. ALTMAN, R.F.A. - A composição química do fruto do "Umary" (Poraqueiba paraensis, Ducke) e (P. sericea, Tul). (Nota preliminar). Manaus, 1956.

5. PROCE, L.T. - Uma expediçăo geológica no alto rio Juruá, Território Federal do Acre, realizada sob os auspicios do Instituto $\mathrm{Na}$ cional de Pesquisas da Amazônia em 1956. Manaus, 1957.

6. RUELLAN, F. - Nota sobre a jazida de calcáreo da Regiẫo do rio Maués. Manaus, 1957.

7. REIS, A.C.F. - Instituto Nacional de Pesquisas da Amazônia. Origem. Objetivos. Funcionamento: Sua contribuição para c conhecimento realístico da Amazônia. Manaus, 1957.

8. RODRIGUES, W.A. - Lista dos nomes vernhiculos da flora do Território do Rio Branco. Manaus, 1957.

9. MOURÃO, O.H. - Estudo sobre medidas das grandezas físicas; precisão de medidas e regras de cálculo. Manaus, 1958.

10. CERQUEIRA, N.L. - Relatório da viagem de reconhecimento ao rio Manacapuru. Manaus, 1958. 
11. REIS, A.C.F. - A expansão portuguesa na Amazônia nos téculos XVII e XVIII. Manaus, 1958.

12. GESSNER, F. - Relatório provisório de pesquisas limnológicas na Região Amazônica, out. 1956/jan. 1957. Manaus, 1958.

13. REIS, A.C.F. - Relatório do INPA, apresentado ao $\mathrm{CNPq}$ relativo ao ano de 1957. Manaus, 1958.

14. BATISTA, D. - Relatório preliminar da expediçăo a Codajás. Manaus, 1958.

15. REIS, A.C.F. - Prestação de conta de uma administração. Manaus, 1958. (Publicação avulsa, 15) (mimeografada).

16. UCHŌ, J. - Atualidades geográficas. Manaus, 1958.

17. UCHOি, J. - Atualidades geográficas (2." série). Manaus, 1958

18. UCHOA, J. - Prontuário geral da divisão territorial, admintsrativa e judiciária do Estado do Amazonas (1957/1961). Manaus, 1959.

10. UCHÔA, J. - Atualidades geográficas (3." série). Manaus, 1959.

20. RODRIGUES, W.A. - Relatório de viagem de reconhecimento dos rios Uatumã e Urubu. Manaus, 1960.

21. UCHÔA, J. - Atualidades geográficas (4." série). Manaus, 1960.

22. MOURÃO, O.H. - Estado de espectro do hidrogênio - cálculo da constante de Plank. Manaus, 1963

23. MIRANDA, P. - Relatório do estágio de limnologia realizado no INPA no período de 4 de jan. a 24 de fev. de 1962. Manaus, 1963.

24. RODRIGUES, W.A. - Situação das investigaçōes florestais do (INPA). Instituto Nacional de Pesquisas da Amazônia. Manaus, 1963.

\section{BOLETIM DO INPA: Série Botânica}

30. SILVA, M.F. da - Estudo sobre Caryocaraceae. II. Contribuição para o conhecimento da morfologia foliar de Caryocar villosum (Aubl.) pers, e Caryocar pallidum A.C. Smith da Amazônia. Manaus, 1969.

31. LOUREIRO, A.A. - Contribuicão ao estudo anatômico da madeira de anonáceas da Amazônia. 1) Unonopsis guatterioides (A.D.C.) R.E. Fries, Fusaea lonqifolia (Aubl.) Saff., Xylopia aromatica Baill. e Ro!linia insignis R.E. Fries var. pallida R.E. Fries. Manaus, 1969.

32. PABST, G.F.J. - Orquídeas novas na região Amazônica (Additamenta ad orchidologiam brasiliensem - VII). Manaus, 1969.

33. ALBUQUERQUE, B.W.P. de - Contribuição ao estudo da nervação foliar de plantas da flora amazônica. ó. Gênero Fagara (Rutaceae). Manaus, 1969.

34. RODRIGUES, W.A. - II. Novos dados sobre a acidez das madeiras da Amazônia. Manaus, 1969.

BOLETIM DO INPA: Série Patologia Tropical; 1970

1. ALMEIDA, F.B. de - Flebótomo da Amazônia. II. Sobre ocorrência de anomalias na genitália masculina em Lytzomya rorotaensis (Floch e Abonnenc 1944) (Diptera, Psychodidae). Manaus, 1970.

2. CERQUEIRA, N.L. - Simuliidae da Amazônia. V. Descriçăo de Simulium septentrionale sp. n. (Diptera, Nematocera). Manaus, 1970.

3. ALMEIDA, F.B. de - Flebótomos da Amazônia. 1. Sobre a presença de Lutzomya anduzei (Rozeboon, 1942) no Brasil (Diptera, Psychodidae). Manaus, 1970.

4. ALMEIDA, F.B. de - Plasmodium brasilianum reencontrado em seu hospedeiro original, o macaco nacari branco, Cacajao calvus. Manaus, 1970.

BOLETIM DO INPA: Série Pesquisas Florestais; 1970

1. BRINKMANN, W.L.F. - Direct and scattered reflection as an aspect of soil survey on the Manaus - Caracaraí - Roadside. Manaus, 1970 ,

2. BRINKMANN, W.L.F. - Neutron scattering and gamma-ray absorption in soil moisture and soil density determination. Manaus, 1970.

3. BRINKMANN, W.L.F. - Optical characteristics of tropical tree-leaves. 1. Andiroba (Carapa guianensis Aubl.). Manaus, 1970.

4. ARAÚJO, V.C. de - Fenologia de essências florestais amazônicas I. Manaus, 1970.

5. HONDA, Mario - Contribuição ao estudo do lenho do gênero Vochysia da Amazônia Brasileira. I. Vochysia guianensis Aubl., V. obscura Warm. e V. inundata Ducke Manaus, 1970.

6. BRINKMANN, W.L.F. - Optical characteristics of tropical tree-leaves. II. Mogno (Swietenia macrophylla King). Manaus, 1970.

7. SANTOS, U. de M. - Método para investigaçōes comparativas sobre materiais húmicos nas águas naturais da Região Amazônica. Manaus, 1970.

8. ALBUQUERQUE, B.W.P. de - Uma nova espécie de Rutaceae da Amazônia. Manaus. 1970.

9. BRINKMANN, W.L.F. - Optical characteristics of tropical tree-leaves and barks. III. Cardeiro (Scleronema micranthum Ducke). Ucuúbas (Virola ssp.), Breus (Protium ssp.) and Matá-Matás (Eschweilera ssp.). Manaus, 1970.

10. MATOS, F.J. de A. - A química de leguminosas brasileiras. XXIV. Os flavonoides da Dalbergia ecastophylla. Manaus, 1970. 
11. CORREA, R.G.C. - O óleo essencial da mirtácea Pseudocaryophyllus pabstianus. Manaus, 1970.

12. ARAÚJO, V.C. de - Sobre a germinaçăo de Aniba (Lauraceae). II. Aniba canelilia (H.B.K.) mez casca preciosa. Manaus, 1970.

13. AlbuOUEROUE, B.W.P. de - Contribuição para o conhecimento das espécies amazônicas do gênero Fagara (Rutaceae). Manaus, 1970.

14. VIEIRA, A.N. - Aspectos silviculturais do "Pau-Rosa" (Aniba Duckei Kostermans). I. Estudos preliminares sobre o incremento volumétrico. Manaus, 1970.

15. LOUREIRO, A.A. - Contribuição ao estudo anatômico da madeira de anonáceas da Amazônia. II. Bocageopsis multiflora (Mart.) R.E. Fries. Guatteria scytophylla Diels Xylopia benthami R.E. Fries e Guatteria olivacea R.E. Fries. Manaus, 1970.

16. HONDA, Mario - Contribuição ao estudo do lenho do gênero Vochysia da Amazônia Brasileira. II. Vochysia maxima Ducke, V. vismifola Spruce ex Warm e V. rufa Mart. Manaus, 1970

17. BRINKMANN, W.L.F. - Relative light intensity measurements in a secondary forest (capoeira) near Manaus - Amazonas - Brazil. Manaus, 1970.

BOLETIM DO INPA: Série Tecnologia

1. MOURÃO, O.H.B. - Determinação do teor de óleo de tungue em mistura com óleo de linhaça.

CADERNOS DA AMAZONIA; 1964 -1966

1. BLUNTSCHLI, Hans - A Amazônia como organismo harmônico. Manaus, 1964.

2. ACKERMANN, F.L. - Geologia e fisiografia da região Bragantina (Estado do Pará). Manaus, 1964.

3. MONTEIRO, M.Y. - O sacado (morfo-dinâmica fluvial). Manaus, 1964.

4. BATISTA, Djalma - Da habilidade da Amazônia Manaus, 1964.

5. MARLIER, Georges - Étude sur les lacs de I'Amazonie Centrale. Manaus, 1965.

6. MONTEIRO, M.Y. - Antropogeografia do guaraná. Manaus, 1965.

7. PINTO, J.M. - Aspectos econômicos da juta na Amazônia. Manaus, 1966.

8. PINTO, O.M. de O. - Estudo crítico e catálogo remissivo das aves do Território $\mathrm{Fe}$ deral de Roraima. Manaus, 1966.

9. MENDES, J.C. - Moluscos da formação Itaituba (Neocarbonifero). Estado do Pará, Brasil. Manaus, 1966.

10. FONTES, G.M.D. de C. - Alexandre Rodrigues Ferreira (aspectos de sua vida e obra) Manaus, 1966.

\section{LIVROS AVULSOS PUBLICADOS PELO INPA}

1. GOODLAND, R. - Glossário de ecologia brasileira. Manaus, Imprensa Oficial do Estado, 1975.

2. PRANCE, G.T. - Árvores de Manaus. Manaus. INPA, 1975. 312p.

3. PRANCE, G.T. - Algumas flores da Amazônia. Manaus, INPA, 1976. 56p.

4. SILVA, M.F. da - Nomes vulgares de plantas amazônicas. Belém, INPA, 1977. 222p.

5. CAVALCANTE, P.S. - Frutas comestiveis da Amazônia. 3.ed. Belém, INPA, 1976. 166p.

6. LOUREIRO, A.A. - Catálogo de madeiras da Amazônia. Belém, SUDAM, 1968, 2v.

7. GOULDING, M. - Ecologia da pesca do rio Madeira. Manaus, INPA, 1979. 172p.

8. SMITH, N.J.H. - A pesca do rio Amazonas Manaus, INPA, 1979. 195p

9. TOKARNIA, C.H. - Plantas tóxicas da Amazô. nia a bovinos e outros herbivoros. Manaus, INPA, 1979. 95p.

10. JUNK, W.J. - Macrófitas aquáticas nas vârzeas da Amazônia e possibilidades do seu uso na agropecuária. Manaus, INPA, 1979. 24p

11. LOUREIRO, A.A. - Essências madeireiras da Amazônia. Manaus, INPA, 1979. 2v.

12. MELO, L.A.S. - Potencialidade agrícola das terras da Amazônia Ocidental. Manaus, SUFRAMA/INPA/EMBRAPA, 1979, 128p.

13. PAHLEN, A von der - Introduçăo à horticultura e fruticultura no Amazonas. Manaus, INPA, 1979. 140p.

14. SCHUBART, H.O.R. - Critérios ecológicos para o desenvolvimento agricola das terras firmes da Amazônia. Manaus, INPA, 1979. 32p.

15. SUANO DA SILVA, A.F. - Repertório bibliográfico dos pesquisadores do INPA. Manaus. INPA, 1980. 81p.

16. ADONIAS, I. - A cartografia da Regiăo Ama. zônica (catálogo descritivo). Rio de Janeiro, IBGE, 1963. 2v.

\section{ACTA AMAZONICA (")}

Acta Amazonica, 1 (1-3): 1971, Suplemento 1 (duas partes)

Acta Amazonica, 2 (1-3): 1972

Acta Amazonica, 3 (1-3): 1973

Acta Amazonica, 4 (1-3): 1974

Acta Amazonica, 5 (1-3): 1975

Acta Amazonica, $6(1-4)$ : 1976, Suplemento 4

Acta Amazonica, $7(1-4)$ : 1977 . Suplemento 4

Acta Amazonica, $8(1-4): 1978$, Suplemento 7

Acta Amazonica, $9(1-4)$ : 1979, Suplemento 4

Acta Amazonica, 10 (1-4): 1980, Suplemento 2

(*) - Com relaçăo oos trabalhos e respectivos autores, consulte o indice geral do último número de cada ano. 